\title{
CIRCUIT BREAKER TYPE DISCONNECTOR for OVERVOLTAGE PROTECTOR
}

\author{
HITOSHI KIJIMA* ${ }^{*}$ MASAO SHIBAYAMA** \\ Electrical Department \\ Polytechnic University \\ 4-1-1 Hashimotodai Sagamihara 229-1196 Kanagawa \\ JAPAN \\ hkijima@uitec.ac.jp* develop1 @nikko-e.co.jp**
}

\begin{abstract}
A disconnector for an overvoltage protector is equipment that disconnects a circuit when the overvoltage protector may fail in a short circuit mode. The disconnector and the overvoltage protector are connected in series. A fuse or a circuit breaker is normaly used as a disconnector. However there are problems with both methods. A fuse is not recyclable once it goes out. Even a circuit breaker is resetable, a lightning surge current causes a swichgear of circuit breaker to open unnecessary. This malfunction prevents the overvoltage protective function which eliminates a lightning surge current into an instrument. If the problem which a switchgear of circuit breaker comes floating can be solved, even when a lightning surge current flows through it, an instrument can be protected by the overvoltage protector. This paper presents a way of preventing circuit breker type disconnectors from the malfunction.
\end{abstract}

Key-Words : circuit breaker, disconnector, overvoltage protector, short circuit mode , lightning surge

\section{Introduction}

This paper presents a disconnector that conducts a current generated by lightning and that blocks a current from a commercial power supply and an overvoltage protection device combining the disconnector with overvoltage protectors $^{[1]-[4]}$. A disconnector for an overvoltage protector is equipment that disconnects a circuit when the overvoltage protector may fail in a short circuit mode. The disconnector and the overvoltage protector are connected in series. A fuse or a circuit breaker is normaly used as a disconnector. However there are problems with both methods. A fuse is not recyclable once it goes out. Even a circuit breaker is resetable, a lightning surge current causes a swichgear of circuit breaker to open unnecessary. Because conventional circuit breakers can not distingnish between a lightning surge current from an altenative overcurent. This malfunction caused by a lightning surge current prevents the overvoltage protective function which eliminates a lightning surge current into an instrument. If the problem which a switchgear of circuit breaker comes floating can be solved, even when lightning surge current flows through it, an instrument can be protected by the overvoltage protector. We present four ideas of preventing circuit breker type disconnectors from the malfunction.

\section{Basic Technology on Disconnector}

Fig. 1 is a configuration example of an overvoltage protection device 1000 combining the disconnector of the present invention and the overvoltage protectors. Although a disconnector 100 needs to be connected in series to overvoltage protectors 801,802 , and 803 between electric wires 981, 982, and 983 and a earth 890, the order does not affect the performance.

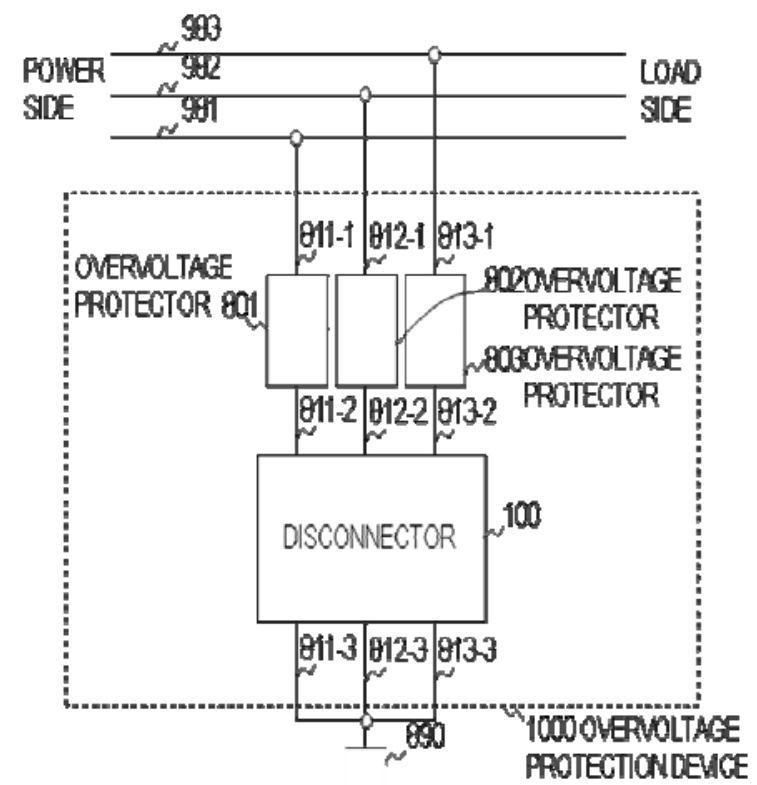

Fig. 1 Configuration example of an overvoltage protection device 
Three electric wires 981, 982, and 983 are wired from the power side to the load side. This is an example of a three-phase AC, and in case of a two-phase three wire AC including neutral wire. The overvoltage protectors 801,802 , and 803 are connected to the electric wires through electric wires $811-1,812-1$, and 813-1. The disconnector 100 is connected to the overvoltage protectors 801,802 , and 803 through electric wires 811-2, 812-2, and 813-2. Furthermore, the disconnector 100 is earthed through electric wires 811-3, 812-3, and 813-3.

When a lightning surge current is generated, the overvoltage protectors are short circuited to protect an instrument instaled on the load side and release the lightning surge current to the earth 890 . Therefore, the disconnector 100 must flow the lightning surge current. Although the lightning surge current is a current that is several $10 \mathrm{kA}$ at the maximum, the maximum current that may flow into the disconnector 100 is small depending on the use environment or the combination with other lightning surge protecting measures. There are various standards for the overvoltage protectors, and the maximum current that the overvoltage protectors can flow is determined by specifications of the overvoltage protectors ${ }^{[1]-[7]}$. Thus, it is preferable that the disconnector 100 can flow the maximum lightning surge current that may possibly flow. However, since the lightning surge current instantaneously (several 10 $\mu$ seconds to several $100 \mu$ seconds) flows, the charge (time integration value of current) is relatively small, which is several coulombs at the maximum.

When there is no more lightning surge current, the overvoltage protectors need to be returned to the disconnected state to flow the commercial power supply to the load side. However, for example, when discharge tubes are used as the overvoltage protectors, once the discharge occurs, the discharge may be continued by the commercial power supply. The phenomenon that the low impedance state of the overvoltage protectors continues even when there is no more lightning surge current and the commercial current flows into the earth 890 is called follow current. The disconnector 100 needs to block the follow current.

Fig. 2 is a diagram showing that the follow current is generated in one overvoltage protector (one-wire earth fault). As shown in Fig. 2, when one overvoltage protector 801 is short-circuited, the current flowing into the earth 890 through the electric wires 811-1 to 811-3 is relatively low. Generally, the current would be several A to several $10 \mathrm{~A}$ because there is earth resistance in the earth 890 .

Meanwhile, as shown in Fig. 3, when both of the overvoltage protectors 801 and 802 are in a low impedance state, i.e. short-circuited, a high current flows because the overvoltage protectors 801 and 802 are short-circuited (interphase short circuit) through the electric wires 981 and 982. Generally, the current would be several 100 A to several $1000 \mathrm{~A}$. The disconnector 100 needs to block the current in both cases. However, in case of commercial power supply, since the current continuously flows, the charge (time integration value of current) in either cases of the follow current tends to be larger than the charge flown due to the lightning surge current.

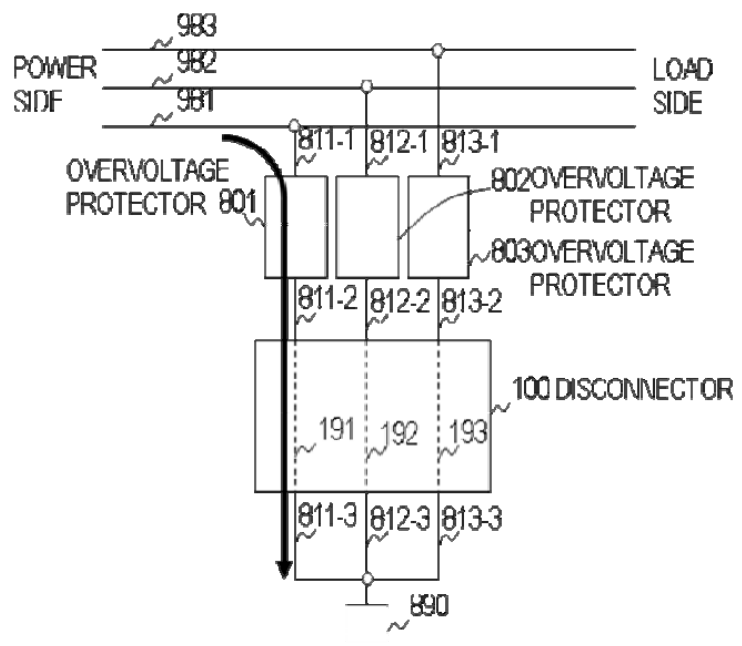

Fig. 2 One-wire earth fault

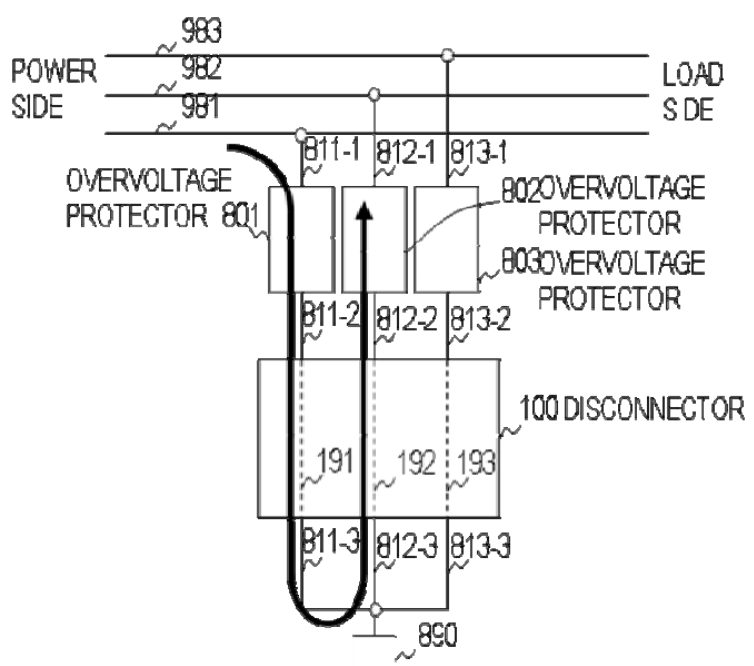

Fig. 3 Two-wire earth fault, i.e., interphase short circuit

Other than the circuit breaker or the fuses, there are residual current devices as means for blocking.

Fig. 4 is a functional configuration example of a residual current device. A residual current device 700 is constituted by a zero phase current transformer (ZCT) 701 that detects the vector sum of the currents flowing through a plurality of electric wires, an amplifier 710 that amplifies an output from the ZCT 701, a switchgear 762 connected to the electric wires in series and capable of disconnecting the electric wires, a trip coil 761 that drives the switchgear 762 , a switching part 750 that applies a current to the trip coil 761 based on the output from the amplifier 710 . When the follow current occurs in one overvoltage protector (in case of one-wire earth fault), the ZCT 701 of the residual current device 700 can detect even a low current. When the low 
impedance state occurs in two or more overvoltage protectors (in case of two-wire earth fault or three-wire earth fault), two or three electric wires are short circuited through the connection to the same earth 890 (interphase short circuit). In case of the interphase short circuit, the directions of the currents of two or three electric wires are opposite. In such a case, since the currents cancel each other, the ZCT 701 cannot detect the follow current. Therefore, the residual current device cannot be used as a disconnector for the overvoltage protection device.

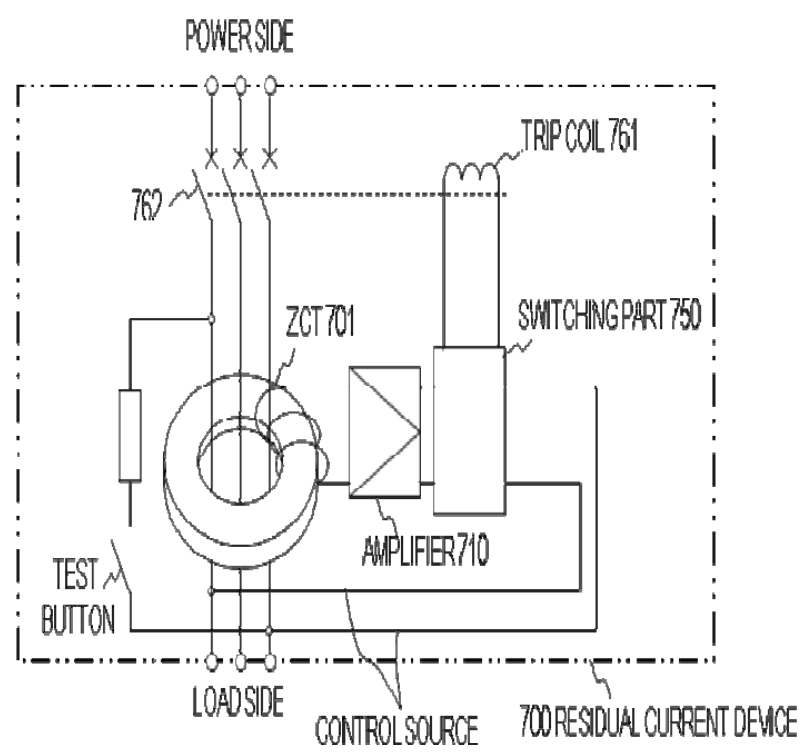

Fig. 4 Functional configuration example of a residual current device

\section{Problem Solutions \\ 3.1 General Ideas}

An object of the present invention is to provide a disconnector that satisfies flowing a lightning surge current, blocking a current from a commercial power supply both in cases of earth fault and interphase short circuit, and being compact in size and an overvoltage protection device combining the disconnector with overvoltage protectors.

(1) A disconnector of the present invention comprises: a current transformer (CT) that detects a current flowing through electric wires; a rectifying part that rectifies the current outputted from the current transformer; a charging part that charges the current outputted from the rectifying part; a switching part that disconnects the electric wires upon driving; and a trigger part that drives the switchgear when the charge stored in the charging part becomes a predetermined drive area. In this regard, the drive area is set larger than the charge that can be stored in the charging part by a lightning surge current. The "charge that can be stored in the charging part by the lightning surge current" denotes a charge multiplying the charge that is determined by the maximum lightning surge current that may flow where the disconnector of the present invention is used or that is determined by the maximum lighting surge current that the overvoltage protectors used in combination with the disconnector of the present invention can handle, by the current transformation ratio of the current transformer.

(2)In the current transformer, when the current flowing through the electric wires is in a range that the trigger part drives the switchgear, the relationship between the current and the voltage outputted when all circuit parts connected to the current transformer are load impedance may be nonlinear. The switchgear may be operated by a current that discharges the charge stored in the charging part. The disconnector may comprise a ZCT that detects the vector sum of the currents flowing through a plurality of electric wires and a bypass part that can set a state that the charge is not stored in the charging part when there are a plurality of electric wires, and may also comprise a current vector sum detecting part that puts the bypass part in a state in which the charge is not stored in the charging part when the output of the ZCT is in a predetermined range. The disconnector may also comprise a delay part that delays the start of charging of the charging part. .

(3)According to the disconnector of the present invention, the lightning surge current is flown because the drive area where the trigger part drives the switching part is set to an area larger than the charge that can be stored in the charging part by the lightning surge current. The current of the commercial power supply can be blocked both in cases of earth fault and interphase short circuit because the charging part can store the current from the continuously flowing commercial power supply.

(4)The current transformer can be miniaturized because the current transformer can be used in the nonlinear region.

(5) Furthermore, the disconnector of the present invention is not affected by the voltage variation of the commercial power supply due to the lightning surge superposition or the like because the disconnector is not supplied with a power supply from the commercial power.

\subsection{First Idea}

Fig. 5 shows a functional configuration example of a disconnector of the first idea.

The disconnector 100 is constituted by: electric wires 191, 192, and 193 for connection with external electric wires; current transformers (CT) 101, 102, and 103 that detect current flowing through the electric wires 191, 192, and 193; a rectifying pat 110 that rectifies current outputted from the CTs 101, 102, and 103; a charging part 120 that charges current outputted from the rectifying part 110; a switching part 140 that disconnects the electric wires upon driving; and a trigger part 130 that drives a switchgear when the charge stored in the charging part becomes a predetermined drive area. In this regard, the drive area is set larger than the charge that can be stored in the charging part 


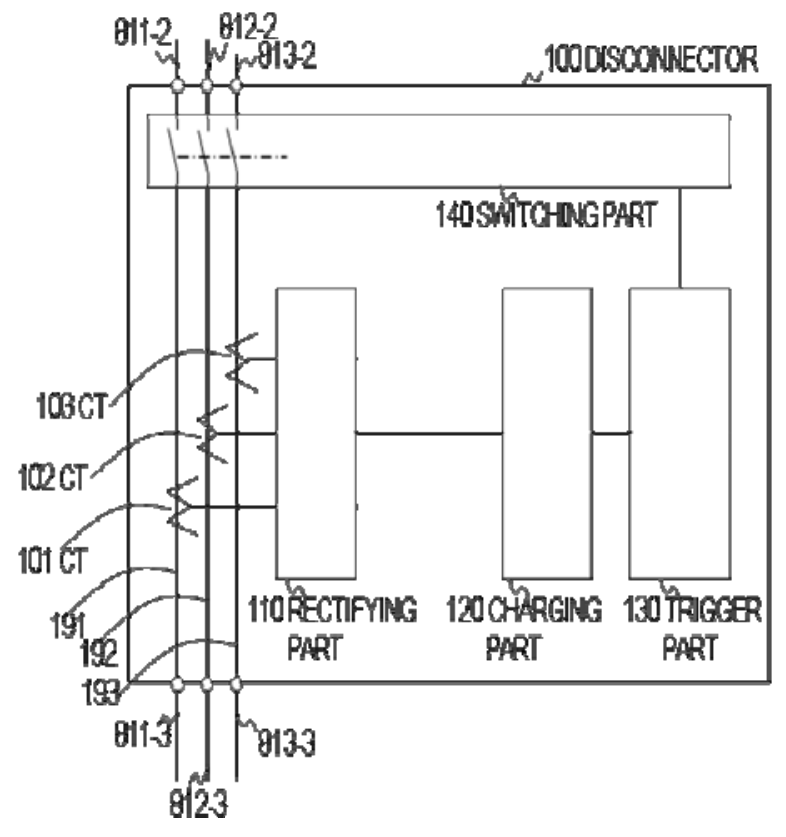

Fig. 5 Functional configuration example of a disconnector of the first idea.

by the outputted voltage when all circuit parts connected to the current transformers (CT) under the lightning surge current are load impedance. The "charge that can be stored in the charging part by the voltage outputted by the current transformers (CT) under the lightning surge current" is provided by a time integration of current flowing through the charging part by the voltage in the linear region or the nonlinear region outputted by the current transformers (CT) 101, 102, and 103 under the lightning surge current. The lightning surge current is determined by, for example, standards ${ }^{[3]-[4]}$ of the maximum lightning surge current that may flow where the disconnector 100 is used or a specification of the maximum lightning surge current of the overvoltage protectors 801, 802, and 803.

Fig. 6 shows a waveform (temporal change) of the lightning surge current.

Since the waveform of the lightning surge current is usually a waveform shown in Fig. 6, the charge can be estimated to some extent once the maximum lightning surge current is determined. In the worst conditions, the maximum current is about $50 \mathrm{kA}$, and the wave tail length is several $100 \mu$ seconds. The maximum current is about 10 $\mathrm{kA}$ in some of the specifications of the overvoltage protectors. In such overvoltage protectors, the overvoltage protectors are broken when the surge current of several 10 kA. Therefore, when thinking about the performance of the disconnector 100 , the performance significantly exceeding the specifications of the overvoltage protectors is not necessary.

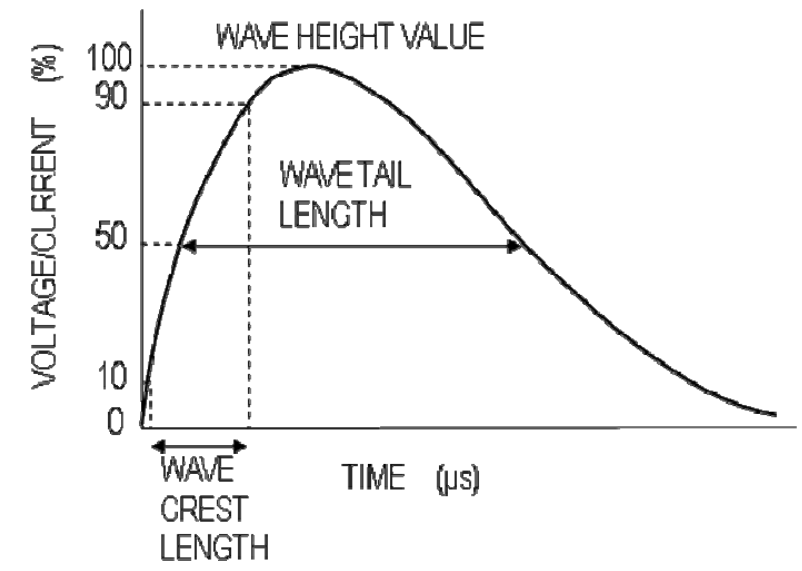

Fig. 6 Waveform of the lightning surge current

Fig. 7 is a diagram showing a specific configuration example of the disconnector 100 . The rectifying part 110 is constituted by diodes 111,112 , and 113 and resistors 114 , 115 , and 116 . The charging part 120 is constituted by a capacitor 121 . The trigger part 130 is constituted by a capacitor 131, resistors 132 and 133, and a thyristor 134 .

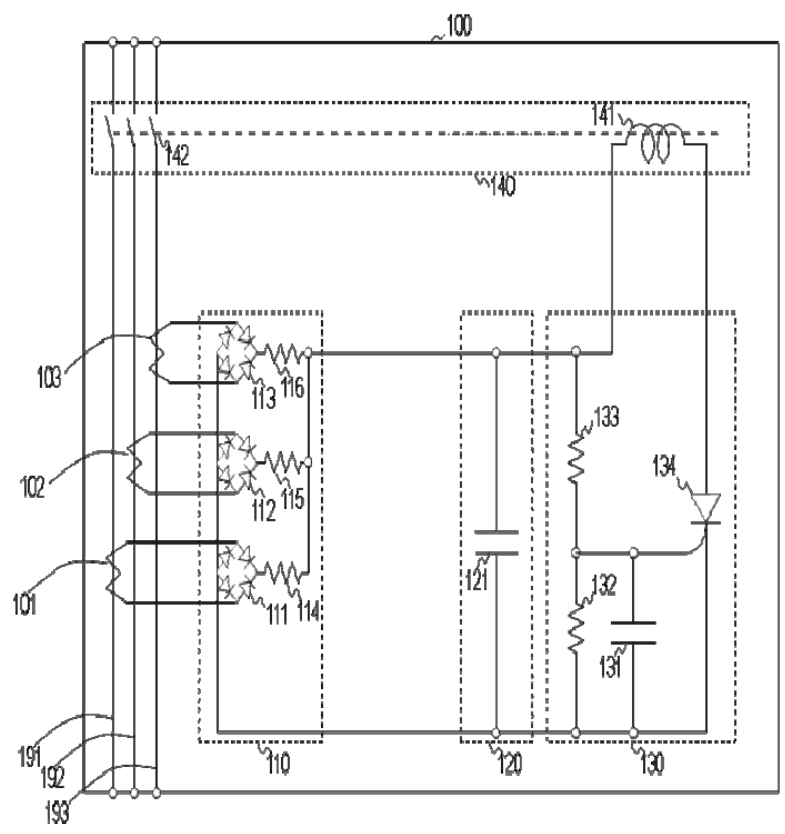

Fig. 7 Specific configuration example of the disconnector 100 .

The switching part 140 is constituted by a trip coil 141 and a switchgear 142. The lightning surge current, the follow current, or the sum of the lightning surge current and the follow current that is detected by the CTs 101, 102, and 103 and that flows through the electric wires 191, 192, and 193 is rectified and charged to the capacitor 121 of the charging part 120 . However, the output voltage from the rectifying part 110 is equal to or lower than the maximum 
voltage generated by any of the CTs 101, 102, and 103. Furthermore, the maximum value of the voltage of the capacitor 121 is the output voltage from the rectifying part 110. Thus, the charge stored in the capacitor 121 is also equal to or lower than the product of the capacity of the capacitor and the maximum voltage that any of the CTs 101, 102, and 103 can output. The input voltage to the trigger part 130 is the voltage of the capacitor 121 . The capacitor 131 stores charge in a voltage lower than the capacitor 121 determined by the ratio between the resistors 132 and 133 . If the voltage generated in the capacitor 131 by the stored charge becomes higher than the drive voltage of the thyristor 134, the thyristor 134 becomes an ON state (short circuit state). The charge stored in the capacitor 121 flows through the trip coil 141, and the magnetic field generated by the trip coil 141 opens the switchgear 142. The follow current is blocked according to such a procedure. A voltage $\mathrm{V}_{131}$ generated in the capacitor 131 is expressed by the following formula.

$$
V_{131}=V_{121} \cdot \frac{R_{132}}{R_{132}+R_{133}}
$$

$\mathrm{V}_{121}$ denotes a voltage of the capacitor $121, \mathrm{R}_{132}$ denotes a resistance value of the resistance 132 , and $R_{133}$ denotes a resistance value of the resistance 133 . After defining that the "charge that can be stored in the charging part (capacitor 121) by the lightning surge current" is Q, the capacitance of the capacitor 121 is C, and the voltage of the capacitor 131 that turns on the thyristor 134 is $S_{131}$,

$$
S_{131}>\frac{Q}{C} \cdot \frac{R_{132}}{R_{132}+R_{133}}
$$

designing the capacity of the capacitor 121 and the resistance values of the resistor 132 and the resistor 133 so as to satisfy the formula enables to design not to turn on the thyristor 134 by the "charge that can be stored in the charging part by the lightning surge current". Designing this way enables to flow the lightning surge current and block the follow current.

Since the disconnector 100 operates in a procedure as described above, sufficient current needs to be applied to the trip coil 141 to surely operate the switchgear 142 . To apply sufficient current to the trip coil 141, a sufficient amount of charge needs to be stored in the capacitor 121 . To store a sufficient amount of charge in the capacitor 121 , it is better that the voltage generated in the CT be larger. It is sufficient if the peak value of the output voltage is increased. Thus, operation in the nonlinear region is possible, and the peak voltage can be utilized after miniaturizing the CT (miniaturizing the core). Therefore, both effects of increasing the output voltage from the CT and miniaturizing the CT can be obtained.
The maximum value of the voltage of the capacitor 121 is an output peak voltage $\mathrm{V}_{\mathrm{CT}}$ of CT. Therefore, when the output peak voltage $V_{\text {Ст }}$ of CT satisfies the following formula, the voltage of the capacitor 131 does not rise up to the voltage that can operate the thyristor 134 .

$$
V_{C T}<S_{131} \cdot \frac{R_{132}+R_{133}}{R_{132}}
$$

(3)

Thus, even if there is a follow current in a range satisfying this formula, the disconnector 100 cannot block the follow current.

For example, a coil having a silicon steel sheet core 20 $\mathrm{mm}$ in dimension and having 3000 turns is used for the CT. The capacitance value of the capacitor 121 is set to $17 \mu \mathrm{F}$, the capacitance value of the capacitor 131 is set to $0.47 \mu \mathrm{F}$, the resistance value of the resistor 132 is set to $4.7 \mathrm{k} \Omega$, and the resistance value of the resistor 133 is set to $2.2 \mathrm{k} \Omega$. Such a disconnector 100 does not operate in a $15 \mathrm{kA}$ lightning surge current, and it is verified that 2 A or higher follow current can be blocked. Short circuit breaking of about 10 $\mathrm{kA}$ is also possible. Furthermore, the CT can be miniaturized. In this example, $15 \mathrm{kA}$ lighting surge can be handled. Furthermore, the disconnector of the present invention can even block a follow current of about 2 A.

\subsection{Second Idea}

Fig. 8 shows a functional configuration example of a disconnector of the second idea.

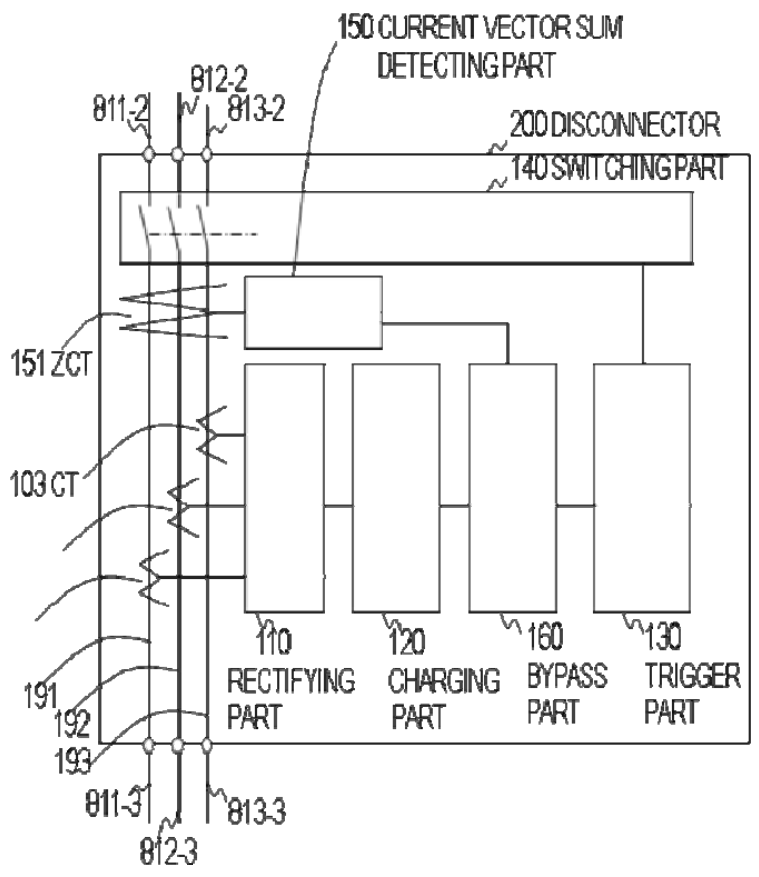

Fig. 8 Functional configuration example of a disconnector of the second idea. 
A disconnector 200 is constituted by adding a zero phase current transformer (ZCT) 151, a current vector sum detecting part 150, and a bypass part 160 to the disconnector 100 (first idea) shown in Fig. 5. The ZCT 151 detects the vector sum of the currents flowing through the electric wires 191, 192, and 193. The vector sum of the currents is a sum including the directions. For example, if the same current is flown through the electric wires 191 and 192 in the opposite directions, the vector sum is 0 (the same as the current is not flowing). Therefore, if a current that only flows on the earth side, such as a lightning surge current, is flown, a high output voltage appears at the output terminal of the ZCT 151. There is almost no output voltage of the ZCT in case of the follow current due to the interphase short circuit shown in Fig. 3. Since the current originally flowing is low in case of the earth fault shown in Fig. 2, the output voltage is low that is clearly different from the lightning surge current. The bypass part 160 bypasses the current outputted from the rectifying part 110 so that the charge is not stored in the charging part 120 . The current vector sum detecting part 150 puts the bypass part 160 into a state that the charge cannot be stored in the charging part 120 if the output voltage of the ZCT 151 is in a predetermined range. The predetermined range is a range that the output voltage from the ZCT 151 can be clearly determined as a lightning surge current. Because of such a functional configuration, a high voltage is outputted from the ZCT 151 when a large current flows only on the earth side, such as a lightning surge current. The current vector sum detecting part 150 sets up the bypass part 160 to bypass the current outputted from the rectifying part 110 . Therefore, the charge is not stored in the charging part 120, and the switching part 140 does not disconnect the electric wires. On the other hand, in case of the follow current caused by the interphase short circuit shown in Fig. 3 or in case of the earth fault shown in Fig. 2, the output voltage from the ZCT 151 is low. Therefore, the current vector sum detecting part 150 sets up the bypass part 160 not to bypass the current outputted from the rectifying part 110. In this state, the current outputted from the rectifying part 110 is stored in the charging part 120, and if the stored charge exceeds a certain value, the trigger part 130 operates the switching part 140 to disconnect the electric wires.

Fig. 9 is a diagram showing a specific configuration example of the disconnector 200.

The current vector sum detecting part 150 is constituted by a diode 151 and resistors 152, 153, and 154. The bypass part 160 is constituted by a transistor 161 and a resistor 162 .

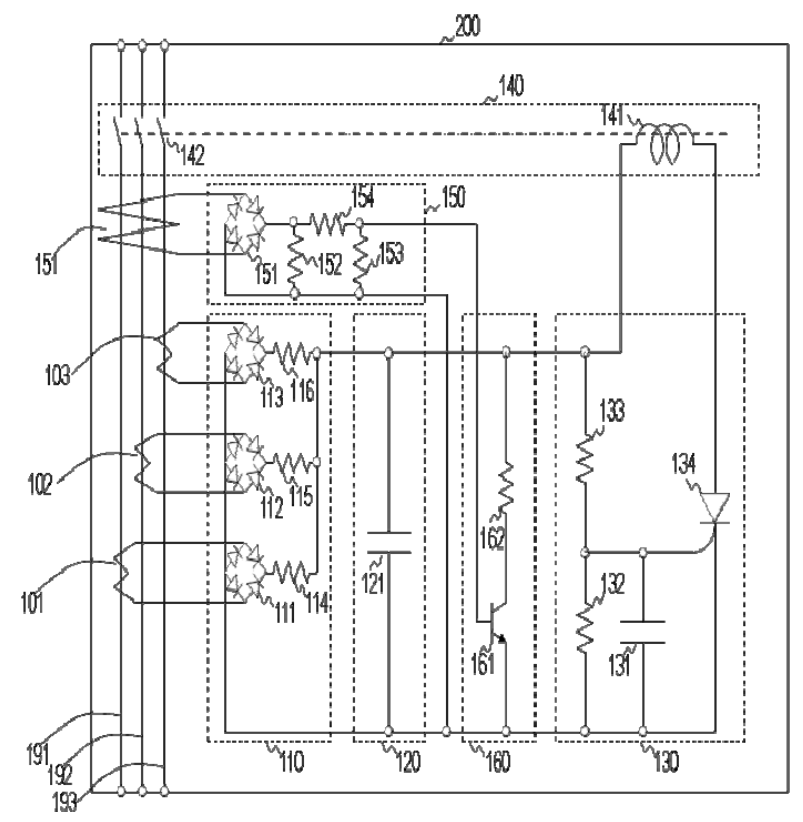

Fig. 9 Specific configuration example of the disconnector 200.

There is no or little output voltage from the ZCT 151 when there is no lightning surge current, so the output voltage from the current vector sum detecting part 150 is low. Therefore, the transistor 161 is OFF, and the current does not flow through the bypass part 160 . Thus, the output current from the rectifying part 110 generated by the follow current is stored in the charging part 120 . When the lightning surge current flows, the output voltage of the current vector sum detecting part 150 becomes higher than the operating voltage of the transistor 161. The transistor 161 then turns on, and the current flows through the bypass part 160. Therefore, the output current from the rectifying part 110 generated by the lightning surge current is hardly stored in the charging part 120 . Thus, in the present idea, the value of Q (charge that can be stored in the charging part by the lightning surge current) is very small. In case of the disconnector 100 (first idea), because the charge generated by the lightning surge current is also stored in the capacitor 121 , the capacitance value of the capacitor 121 needs to be enlarged to some extent. However, if the capacitance value of the capacitor 121 is enlarged, the time until blocking is long, because the switchgear 142 is not operated until the charge is stored by the follow current. The follow current can be blocked quicker than the first idea because the disconnector 200 can set the capacitance value of the capacitor 121 to the minimum capacitance that the trip coil 141 operates the switchgear 142 . The disconnector 100 also blocks the lightning surge current if the charge that operates the switchgear 142 is stored in the capacitor 121 only by the lightning surge current. Since the bypass part 160 of the disconnector 200 does not store the output current from the CTs 101, 102, and 103 generated by the lightning surge 
current in the charging part 120 , there is no fear that the lightning surge current operates the switchgear 142 .

For example, a coil having a silicon steel sheet core 30 $\mathrm{mm}$ in dimension and having 6000 turns is used for the ZCT. A coil having a silicon steel sheet core $20 \mathrm{~mm}$ in dimension and having 3000 turns is used for the CT. The capacitance value of the capacitor 121 is set to $17 \mu \mathrm{F}$, the capacitance value of the capacitor 131 is set to $0.47 \mu \mathrm{F}$, the resistance value of the resistor 132 is set to $4.7 \mathrm{k} \Omega$, the resistance value of the resistor 133 is set to $2.2 \mathrm{k} \Omega$, and the resistance value of the resistor 162 is set to $6 \mathrm{k} \Omega$. Such a disconnector 200 does not operate under the lightning surge current of $50 \mathrm{kA}$ and can block a 2 A or higher follow current. Short circuit breaking of about $10 \mathrm{kA}$ is also possible.

\subsection{Third Idea}

Fig. 10 shows a functional configuration example of a disconnector of the third idea.

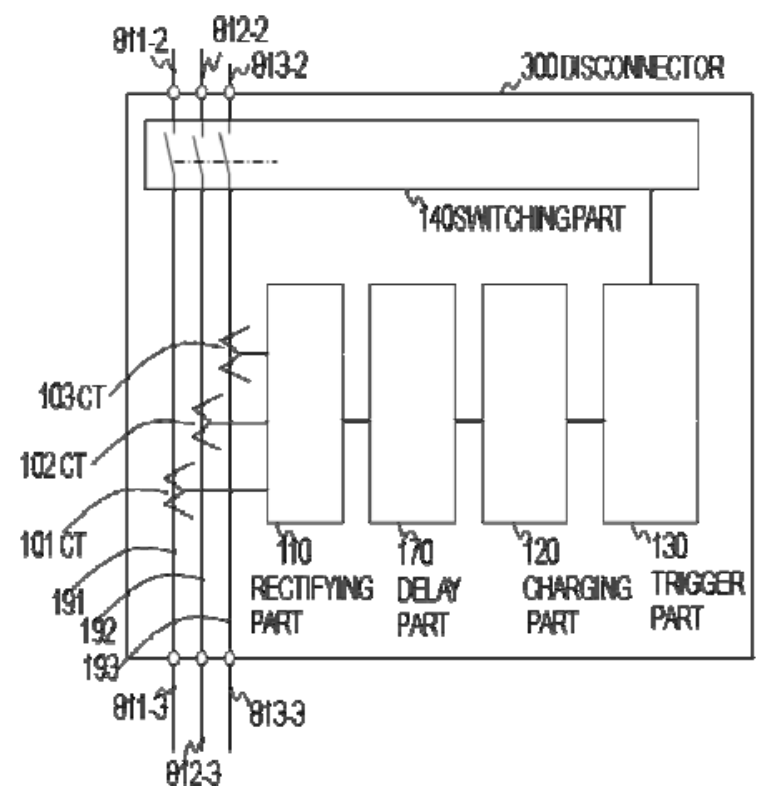

Fig. 10 Functional configuration example of a disconnector of the third idea.

The disconnector 300 is constituted by adding a delay part 170 to the disconnector 100 (first idea) shown in Fig. 5. When the lightning surge current flows, the delay part 170 slightly delays the timing that the charging part 120 starts to charge the current from the rectifying part 110 . In the second idea shown in Fig. 8, the current vector sum detecting part 150 and the bypass part 160 are used to prevent the current from the rectifying part 110 by the lightning surge current from being charged to the charging part 120 . In the present idea, the timing that the charging part 120 starts charging is delayed to prevent the current from the rectifying part 110 by the lightning surge current from being charged to the charging part 120.
Fig. 11 shows a specific configuration example of the disconnector 300 .

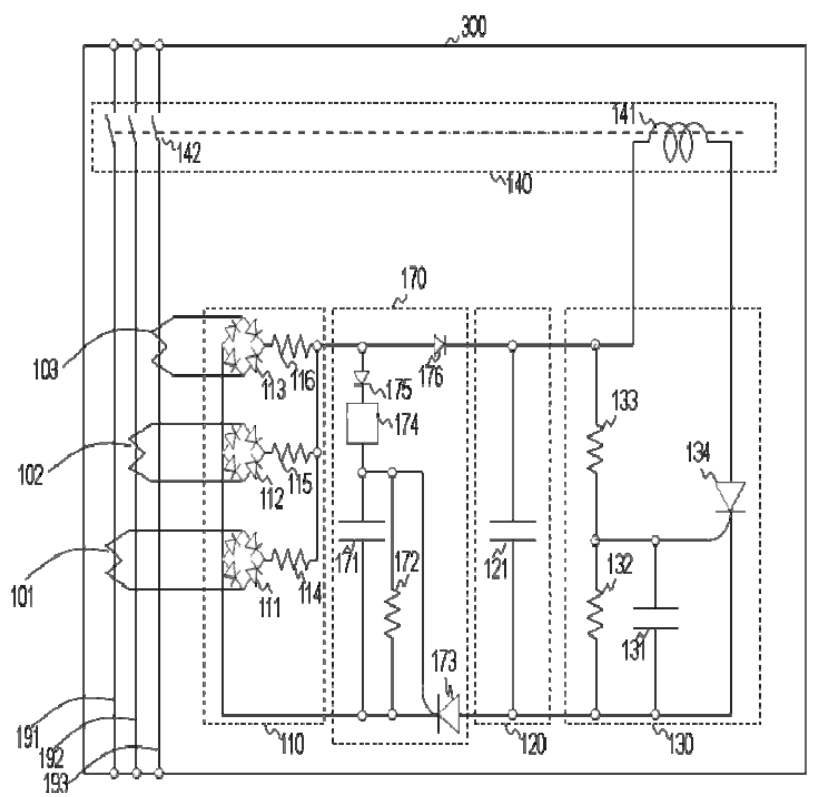

Fig.11 Specific configuration example of the disconnector 300 .

The delay part 170 is constituted by a capacitor 171 , a resistor 172, a thyristor 173 , a current limiting circuit 174 , and diodes 175 and 176 .

Figs. 12A, 12B, and 12C show examples of the current limiting circuit 174. In Fig. 12A, a resistor limits the current. Fig. 12B shows an example of a constant current circuit using a transistor 1742. Fig. 12C is an example using a constant current diode 1746 . .

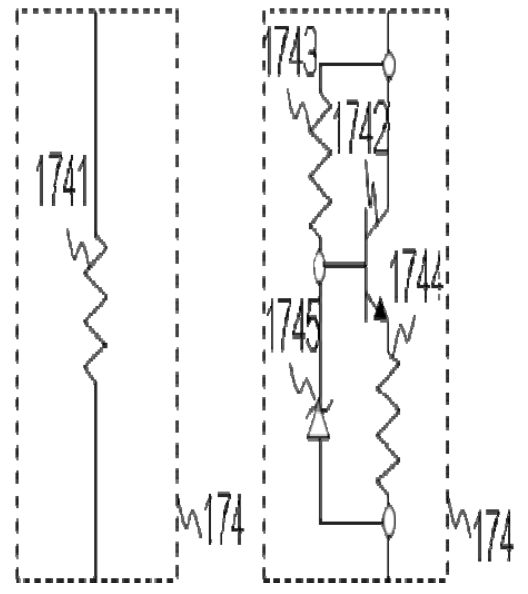

Fig. 12A
Fig. 12B

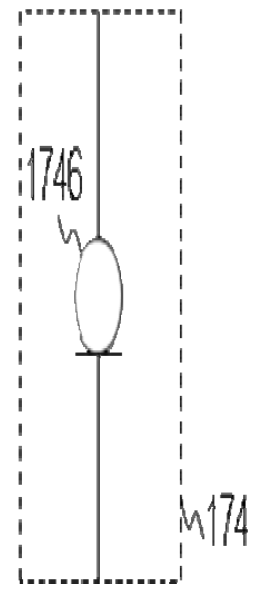

Fig. 12C
Fig. 12 Examples of the current limiting circuit 174 
When the lighting surge is not generated, there is no output current from the rectifying part 110, and the charge of the capacitor 171 is 0 . Therefore, the thyristor 173 is $\mathrm{OFF}$, and the current from the rectifying part 110 does not flow through the charging part 120 . When the lightning surge is generated, the current from the rectifying part 110 is charged to the capacitor 171 through the current limiting circuit 174 . When the voltage of the capacitor 171 exceeds a predetermined value, the thyristor 173 is turned on. The time from the generation of the lightning surge current to the turning on of the thyristor 173 is a delay generated by the delay part 170 . If the current value of the current limiting circuit 174 and the capacitance value of the capacitor 171 are designed so that the delay time is several $100 \mu$ seconds, the current from the rectifying part 110 by the lightning surge current is not charged to the charging part 120. The resistor 172 is arranged to discharge the charge of the capacitor 171 to return the thyristor 173 to the initial OFF state when there is no more lightning surge current. The diodes 175 and 176 are arranged to prevent the backflow.

When the thyristor 173 is turned on, the current from the rectifying part 110 is also charged to the capacitor 121 of the charging part 120. However, the current is not charged to the capacitor 121 if there is no follow current. Therefore, the switching part 140 is not driven. On the other hand, the charge is stored in the capacitor 121 if there is a follow current. When the charge stored in the capacitor 121 exceeds a predetermined value, the trigger part 130 drives the switching part 140, and the electric wires are disconnected. In the present idea, the value of Q (charge that can be stored in the charging part by the lighting surge current) is very small as in the second idea. Therefore, the capacitance $\mathrm{C}$ of the capacitor 121 can also be made small, and the same effects as the second idea can be obtained.

\subsection{Fourth Idea}

Figs. 13A and 13B show specific configuration examples of the switching part 140 .

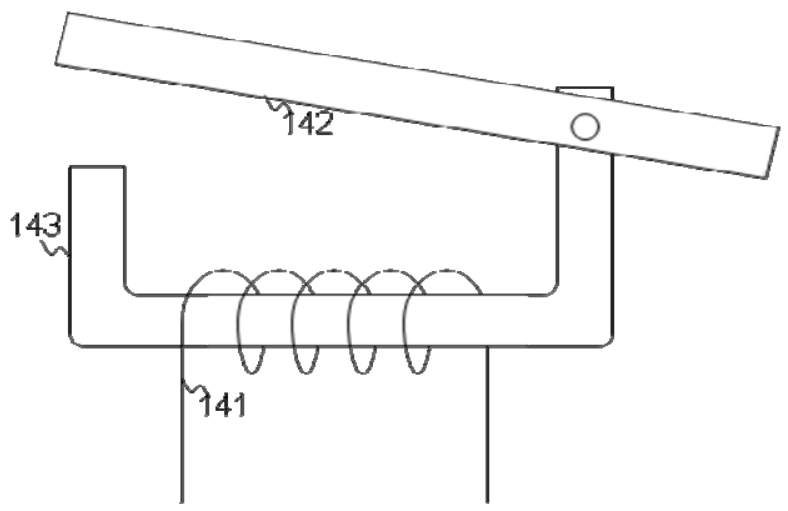

Fig. 13A Specific configuration examples of the switching part 140
The switching part 140 of Fig. 13A is constituted by the trip coil 141, the switchgear 142, and a magnetic frame 143. After a current is applied to the trip coil 141, the magnetic frame 143 works as a magnet and operates the switchgear 142.

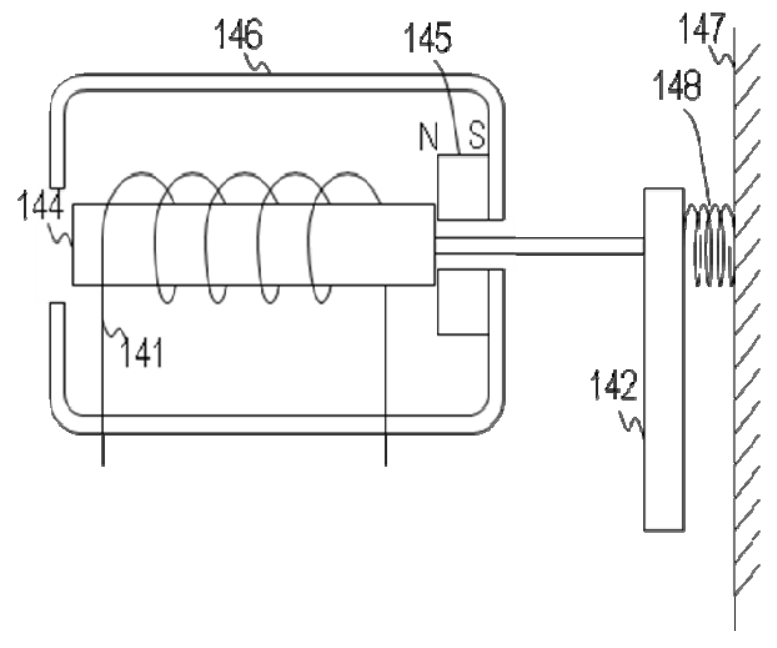

Fig. 13B Specific configuration examples of the switching part 140

The switching part 140 of Fig. 13B is constituted by the trip coil 141, the switchgear 142, an iron core 144 , a permanent magnet 145, a magnetic frame 146, a fixed part 147, and a spring (elastic body) 148. The iron core 144 is connected to the switchgear 142 . When the current is not flowing through the trip coil 141, the iron core 144 is attracted to the permanent magnet 145 . When a current that generates a magnetic field in the opposite direction of the permanent magnet 145 flows through the trip coil 141, the magnetic field is canceled, and the iron core 144 is not attracted to the permanent magnet 145 . At this point, the spring 148 pulls away the iron core 144 from the permanent magnet 145. Actual mounting of the disconnector will now be described. Since the switchgear 142 is driven by the magnetic force generated by the trip coil 141, the switchgear 142 is arranged near the trip coil 141. Since the switchgear 142 is a mechanism that opens and closes the electric wires 191, 192, and 193, the switchgear 142 is arranged near the electric wires 191, 192, and 193. Therefore, the trip coil 141 is also arranged near the electric wires 191, 192, and 193. Furthermore, the constituting parts of the disconnector are densely mounted for miniaturization. Because of the arrangement, when a current flows through all or part of the electric wires 191, 192, and 193 by the lightning surge and then the magnetic field is generated, the trip coil 141 is exposed in the magnetic field. In case of the switching part of Fig. 13A, the switchgear 142 may open even if the current is not flowing through the trip coil 141. In case of the switching part of Fig. 13B, there would be fewer malfunctions than in the switching part of Fig. 13A, but malfunctions may still occur. Thus, in the present 
invention, the switching part of Fig. 13B is used to produce, in the trip coil 141, a magnetic field in the same direction as the permanent magnet 145 when the current flows through all or part of the electric wires 191, 192, and 193 by the lightning surge. Therefore, since the iron core 144 is strongly attracted to the permanent magnet 145 , there is no fear of malfunction.

Fig. 14 shows a functional configuration example of a disconnector of the fourth idea. A disconnector 400 is characterized by using the switching part 140 shown in Fig. $13 \mathrm{~B}$ and adding a reverse exciting part 180 to the disconnector 200 (second idea) shown in Fig. 8. By adding the reverse exciting part 180 , minor changes are made to a rectifying part $110^{\prime}$ and a trigger part 130 '. In addition to the functions of the rectifying part 110, the rectifying part 110' includes a function of supplying a current to the reverse exciting part 180 when the current flows through all or part of the electric wires 191, 192, and 193 by the lightning surge. The reverse exciting part 180 supplies a current to the switching part 140 so that the switchgear is closed. The trigger part 130' is constituted such that the current supplied to the switching part 140 by the reverse exciting part 180 is not inputted.

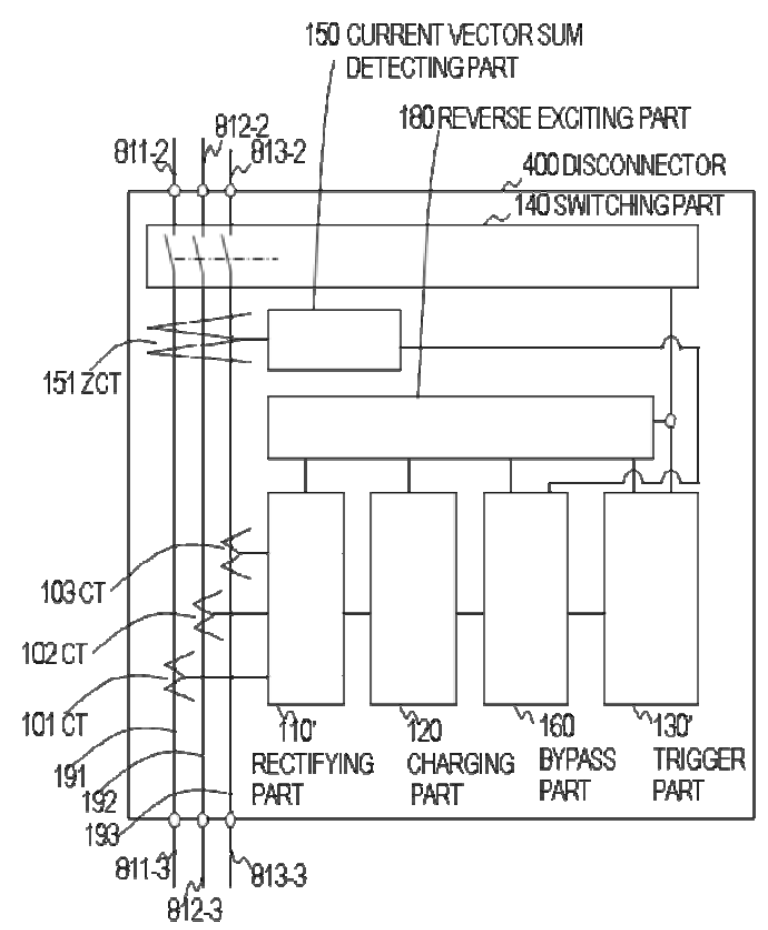

Fig. 14 Functional configuration example of a disconnector of the fourth idea.

Fig. 15 shows a specific configuration example of the disconnector 400 .

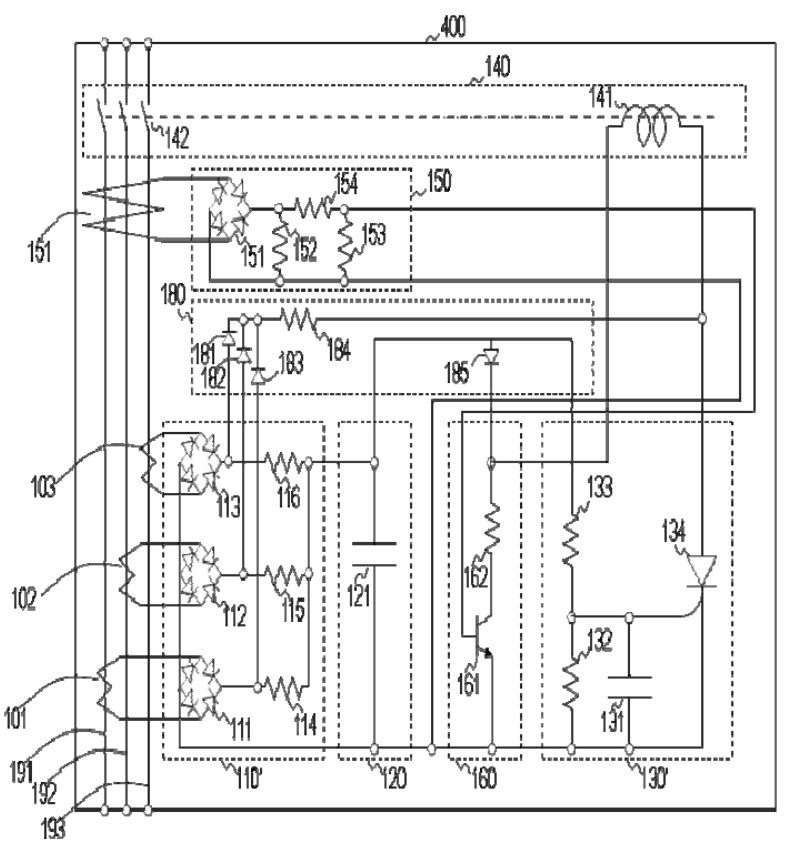

Fig. 15 Specific configuration example of the disconnector 400 .

The reverse exciting part 180 is constituted by diodes 181,182 , and 183 , a resistor 184 , and a diode 185 . The diodes 181, 182, and 183 rectify and add up the current from the rectifying part 110'. The resistor 184 adjusts the amount of current applied to the trip coil 141. The diode 185 is for backflow prevention and allows only the charging part 120 to supply the current to be supplied to the trigger part 130'. The wiring of the rectifying part $110^{\prime}$ is configured to be able to supply, after rectification, part of the current to be supplied to the charging part 120 to the reverse exciting part 180. The wiring of the trigger part 130 ' is configured to supply the current to the resistor 133 only from the charging part 120.

When the lightning surge current flows, the output voltage of the current vector sum detecting part 150 becomes higher than the operating voltage of the transistor 161. The transistor 161 is then turned on. The current flows from the reverse exciting part 180 to the trip coil 141. The direction of the current is opposite the current flowing from the charging part 120 to the trip coil 141 . Therefore, the switchgear 142 can strongly maintain the closed state against the lightning surge current. For example, if the resitance value of resistor 184 is $33 \Omega$ when the reactance value of the trip coil 141 is $30 \Omega$, the switchgear 142 can be closed with a backward current that is more than twice the forward current (current in the direction that the switchgear 142 opens) in a $20 \mathrm{kA}$ lighting surge.

In case of the follow current, since the output voltage of the current vector sum detecting part 150 is lower than the operating voltage of the transistor 161, the current does not flow from the reverse exciting part 180 to the trip coil 141 . If sufficient charge is stored in the capacitor 121 of the 
charging part 120 and the thyristor of the trigger part 130' is turned on, the current flows through the trip coil 141, and the switchgear 142 is disconnected. Such a configuration of the disconnector 400 enables to surely flow the lightning surge current and block the follow current without the switchgear 142 malfunctioning.

\section{Conclution}

This paper presented a disconnector that conducts a current generated by lightning and that blocks a current from a commercial power supply. We proposed four ideas of preventing circuit breker type disconnentors from the malfunction caused by lightning surge current.

(1) First idea;the lightning surge current is flown because the drive area where the trigger part drives the switching part is set to an area larger than the charge that can be stored in the charging part by the lightning surge current. The current of the commercial power supply can be blocked both in cases of earth fault and interphase short circuit because the charging part can store the current from the continuously flowing commercial power supply.

(2) Second idea; the disconnector comprise a ZCT that detects the vector sum of the currents flowing through a plurality of electric wires and a bypass part that can set a state that the charge is not stored in the charging part when there are a plurality of electric wires, and may also comprise a current vector sum detecting part that puts the bypass part in a state in which the charge is not stored in the charging part when the output of the ZCT is in a predetermined range.

(3) Third idea; the disconnector comprise a delay part. When the lightning surge current flows, the delay part slightly delays the timing that the charging part starts to charge the current from the rectifying part.

(4) Fourth idea; the disconnector is adding a reverse exciting part to the disconnector . The reverse exciting part supplies a current to the switching part so that the switchgear is closed when the current flows through all or part of the electric wires by the lightning surge.

The disconnectors mentioned above are not affected by the voltage variation of the commercial power supply due to the lightning surge superposition or the like because the disconnectors are not supplied with a power from a commercial power line.

\section{References:}

[1] H. Kijima "Earthing system and lightning protection,” Corona Co. Published, ISBN4-88552-147-C3055, 2002

[2] H. Kurosawa, H. Kijima "Recent lightning protection design,” JSA (Japanese Standard Association) Published, ISBN4-542-30397-7-C3054, 2006
[3] IEC 61643-1, Surge protective devices connected to low voltage power distribution systems Part 1: Requirements and test methods, 2005

[4] IEC 61643-12, Surge protective devices connected to low voltage power distribution systems Part 12: Selection and application principle, 2004

[5] H. Kijima "Overvoltage protective device and method of overvoltage protection, Japanese patent No.3854305, September 2006

[6]H. Kijima, Overvoltage protective device and method of overvoltage protection, Korean patent No. 10-0845224, July 2008

[7] H. Kijima, Overvoltage protective device and method of overvoltage protection, Australian patent No.2006246468 , October 2008 